This item was submitted to Loughborough's Research Repository by the author.

Items in Figshare are protected by copyright, with all rights reserved, unless otherwise indicated.

\title{
Qualitative investigation of exercise perceptions and experiences in people with multiple sclerosis before, during, and after participation in a personally tailored exercise program
}

\section{PLEASE CITE THE PUBLISHED VERSION}

https://doi.org/10.1016/j.apmr.2017.05.022

\section{PUBLISHER}

Elsevier () American Congress of Rehabilitation Medicine

\section{VERSION}

AM (Accepted Manuscript)

\section{PUBLISHER STATEMENT}

This work is made available according to the conditions of the Creative Commons Attribution-NonCommercialNoDerivatives 4.0 International (CC BY-NC-ND 4.0) licence. Full details of this licence are available at: https://creativecommons.org/licenses/by-nc-nd/4.0/

\section{LICENCE}

CC BY-NC-ND 4.0

\section{REPOSITORY RECORD}

Crank, Helen, Anouska Carter, Liam Humphreys, Nicky Snowdon, Amanda Daley, Nicola Woodroofe, Basil Sharrack, Jane Petty, and John M. Saxton. 2019. "Qualitative Investigation of Exercise Perceptions and Experiences in People with Multiple Sclerosis Before, During, and After Participation in a Personally Tailored Exercise Program". figshare. https://hdl.handle.net/2134/32199. 
Running head: Experiences of exercise in people with MS

A qualitative investigation of exercise perceptions and experiences in people with multiple sclerosis before, during and after participation in a personally-tailored exercise program

Helen Crank $\mathrm{PhD}^{1}$, Anouska Carter $\mathrm{PhD}^{1}$, Liam Humphreys $\mathrm{MSc}^{1}$, Nicky Snowdon $\mathrm{MSc}^{2}$, Amanda Daley $\mathrm{PhD}^{3}$, Nicola Woodroofe $\mathrm{PhD}^{4}$, Basil Sharrack MD, PhD, FRCP, FAAN ${ }^{5}$, Jane Petty $\mathrm{BSc}^{6}$ and John M Saxton $\mathrm{PhD}^{7}$

${ }^{1}$ The Centre for Sport and Exercise Science, Sheffield Hallam University, UK

${ }^{2}$ Centre for Health and Social Care Research, Sheffield Hallam University, UK

${ }^{3}$ Primary Care Clinical Sciences, University of Birmingham, UK

${ }^{4}$ Biomolecular Sciences Research Centre, Sheffield Hallam University, UK

${ }^{5}$ Academic Department of Neurology, Sheffield Teaching Hospitals NHS Foundation Trust, UK

${ }^{6}$ Multiple Sclerosis Society, UK

${ }^{7}$ Department of Sport, Exercise and Rehabilitation, Northumbria University, UK

We acknowledge the support of the UK Multiple Sclerosis Society: Project Grant 888/08

\section{Corresponding author:}

Professor John M Saxton

Faculty of Health and Life Sciences

Northumbria University

Room 259, Northumberland Building

Newcastle Upon Tyne

NE1 8ST

United Kingdom

Tel + 44 (0)1912273371

Email: john.saxton@northumbria.ac.uk

Reprints are not available 
Clinical Trial Registration Number: ISRCTN41541516 


\section{ACCEPTED MANUSCRIPT}

A qualitative investigation of exercise perceptions and experiences in people with multiple sclerosis before, during and after participation in a personally-tailored exercise program

\section{ABSTRACT}

4 Objective: To undertake a qualitative investigation of exercise perceptions and experiences in

5 people with MS (PwMS) before, during and after participation in a personally-tailored program

6 designed to promote long-term maintenance of self-directed exercise. Design: Focus groups and

7 semi-structured telephone interviews. Setting: University Exercise Science Department close to

8 the recruiting hospital. Participants: PwMS (N=33; aged 47.6 \pm 7.9 y). Interventions:

9 Participants were recruited after participation in a randomized controlled exercise trial; all had

10 been allocated to a 12-week exercise programme, comprising supervised and self-directed

11 exercise sessions. Main outcome measure: Exercise perceptions and experiences before, during and after participation in the program. Results: Four themes emerged from the analysis: (1) the transition to inactivity; (2) lack of knowledge and confidence; (3) positive exercise experiences; (4) perspectives on exercise adherence. Conclusion: Lack of confidence and exercise knowledge, coupled with negative perceptions about physical capabilities after an MS diagnosis, are clear barriers to exercise participation in PwMS. These issues are not being adequately addressed as part of the healthcare pathway or in community settings. Perceptions of improved posture, ability to overcome everyday difficulties, acute mood enhancements during and after exercise and increased opportunities for social interaction were amongst the reported benefits of exercise participation. Despite the provision of a personally-tailored exercise plan and use of cognitive behavioural strategies, self-directed exercise continued to present challenges to PwMS and the importance of seeking cost-effective ways to maintain motivational support was implicit in participant responses.

Keywords: Multiple sclerosis, exercise, qualitative evaluation 
INTRODUCTION

28 Studies have shown that exercise is a safe non-pharmacological treatment strategy for people

with multiple sclerosis (PwMS), with the reported health benefits including improvements in muscle power, physical and psychological functioning, fatigue and health-related quality of life. ${ }^{1}$ ${ }^{2} 3$ However, following an MS diagnosis, perceived functional limitations, safety concerns and loss of confidence, fatigue, lack of MS-specific exercise knowledge or conflicting advice from health professionals and accessibility issues associated with inadequate transport, physical environment and social factors (e.g. social obligations, lack of social support) become important barriers to exercise participation. ${ }^{4-10}$ Cross-sectional evidence suggests that only $\sim 20 \%$ of PwMS are achieving recommended amounts of daily moderate to vigorous intensity physical activity ${ }^{11}$ and strategies to address common barriers are needed to increase the level of engagement.

Confidence in managing disease-specific symptoms, as well as positive coping styles, selfcharacteristics of PwMS who continue to be physically active after diagnosis. ${ }^{9,}{ }^{12}$ In addition, the support and knowledge gained from competent health professionals, perceived physical, mental and social benefits, peer support and camaraderie during group exercise, and feelings of accomplishment, self-management and control are important facilitators that lead to improved self-efficacy for exercise. ${ }^{4,8-13,14}$ Despite this, Kayes et $\mathrm{al}^{10}$ concluded that the decision to

47 engage in physical activity is complex, individual and fluid amongst PwMS, reflecting the day-

48 to-day uncertainty of the condition. This suggests a personalised approach to barrier management may be needed. Furthermore, programs which provide support for self-directed exercise, taking into consideration the day-to-day challenges MS symptom management,

51 individual capabilities and personal preferences may be more effective for promoting sustainable 52 physical activity behaviour change. 
54 We recently reported on health and cost utility outcomes following a randomized controlled trial

which investigated a pragmatic approach to developing the skills and confidence for long-term maintenance of self-directed exercise in people with mild to moderate MS (ExIMS). ${ }^{15} 16$ The

57 program provided access to an exercise physiologist and physical therapist, who applied cognitive behavioural techniques during a graded decrease in the frequency of individuallytailored supervised exercise (three exercise sessions per week for 12 weeks, with the ratio of supervised to home-exercise sessions being 2:1 in weeks 1-6 and 1:2 in weeks 7-12). There was months after withdrawal of supervision. ${ }^{15}$

The purpose of this study was to undertake a qualitative investigation of exercise perceptions and experiences in people with MS (PwMS) before, during and after participation in this

67 personally-tailored program designed to promote long-term maintenance of self-directed exercise. An improved understanding of exercise perspectives and personal experiences of engaging with programs that can develop skills to support the self-management of MS will inform future healthcare implementation strategies aimed at enhancing patient care.

\section{METHODS}

\section{Study design}

74 We used qualitative research methods to explore exercise perceptions and experiences. Our

75 underlying philosophy was constructivist, ${ }^{17}$ recognising the individual nature of experience and

76 the impact of people's wider life experiences on their perspectives of exercise both before and

77 during the trial. Data collection used both focus groups and individual interviews. An advantage

78 of focus groups is that social interaction between group members can increase the depth of 
inquiry, stimulating discussion of shared experiences and their meaning to each individual.

80 Conversely, interview responses can provide participants with more opportunity to contribute so using a combination of methods can yield different viewpoints. ${ }^{13}$ In addition, we were keen to understand all participants' experiences; and offering a choice of methods meant that participants unable to join focus groups could organise interviews around their availability.

\section{Participants}

A purposive convenience sample of $54 \mathrm{PwMS}$ who had participated in the 12 week exercise

87 program ${ }^{15}$ were invited to take part in in the qualitative investigation. Of these, 33 participants agreed to participate and were recruited within 6 months of completing the program and their characteristics are presented in Table 1. Written informed consent was obtained prior to study participation and ethical approval was granted from NHS South Yorkshire Research Ethics

91 Committee.

\section{Focus groups and telephone interviews}

94 A total of 29 participants took part in the focus groups and four participants were interviewed by telephone. Six focus groups (2-8 participants; 60-80 min duration) were facilitated by two researchers trained in qualitative research techniques. Facilitators were not directly involved in

97 the exercise trial but do work in exercise research. Telephone interviews ( $\sim 30$ min duration) were conducted by a member of the trial team who had delivered some of the exercise training sessions. All participants were briefed about the purpose of the discussion, i.e. to elicit their views and experiences of exercise and engaging in the program, and the same semi-structured $a$ priori topic guide consisting of open-ended questions was used flexibly to guide the focus group and interview discussions (Table 2). Focus group discussions and telephone interviews were audio-taped and then transcribed verbatim by a source independent of the study. Participant anonymity was assured by the assignment of reference numbers. 


\section{Thematic framework analysis}

107 Using framework analysis, ${ }^{18} \mathrm{HC}$ and LH independently read and re-read the transcripts several 108 times to become familiar with the data, before coding, indexing and charting to create an initial 109 key thematic framework with sub-themes. Analysis aimed at describing the individual's 110 experience of exercise, searching for common, recurrent patterns but also identifying insights 111 into participant experiences that might explain behaviour and improve advice and services in the future. NS and AC read the transcripts and independently applied the coding framework. The coding framework represented all relevant data and there was a high level of agreement between analysers. This approach to data analysis is somewhat deductive, framing the analysis within an a-priori topic guide, yet the data were borne out of original transcripts from focus groups and interviews. ${ }^{19}$ Data saturation was achieved, in that similar themes arose repeatedly and no new themes arose in the final focus group or interview.

\section{RESULTS}

120 The findings are presented under four key themes, which contextualise the experiences of participants in the study. Direct quotes, with reference to either focus group and participant number, or telephone interview and participant number, are presented to illustrate the key themes and sub themes.

Theme 1: The transition to inactivity

126 Prior to MS, many participants had valued the personal, social and health benefits of a

127 physically active lifestyle, however, their MS diagnosis caused a transition into physical inactivity. Several participants recounted how regular exercise was a part of 'normal life' before MS and helped to define their self-identity. Comments included: "I used to play a lot of sport. 


\section{ACCEPTED MANUSCRIPT}

For others, an active lifestyle before MS had been a shared and valued experience with family

and friends, a social activity or something that was done for fitness, recreation and health: "I

became like one of those people who use the swimming pool, jacuzzi, sauna and things like that

on a Sunday morning” (FG1:1).

However, a diagnosis of MS had created a transition toward physical inactivity and a perceived reduction in exercise capacity for many participants: "I would say I became very inactive"

(FG1:2). Other participants attempted to maintain their exercise levels but found it difficult: "I was still a member of a gym but it ended up that I would drive to the gym and then I would be sat in tears in the car thinking I can't do that... so I gave up going to the gym because it is supposed to be there to help me but it was carving me up and making my symptoms worse"

142 (FG1:4). A small minority of participants were able to maintain regular exercise by becoming adept at pre-empting situations, planning ahead and making adaptations to their lifestyle with a positive frame of mind.

Theme 2: Lack of knowledge and confidence

147 There was general consensus that exercise advice from health professionals was either nonexistent or not relevant for the needs of PwMS. Participants recalled that exercise and lifestyle advice was not generally offered after their MS diagnosis: "I don't recall any professionals mentioning anything about exercise at all and I don't believe I asked" (FG5:2). Participants felt that endorsement from the clinical team was needed to confirm that exercise was something you were allowed to and supposed to do, but this advice was variable: "When I was diagnosed I asked specifically if there was anything I could do to help myself, diet wise and exercise and I was told there was nothing you could do whatsoever" (FG2:6). Other participants were referred to local facilities but the tailored exercise advice and support they needed was lacking: " $M y$ 


\section{ACCEPTED MANUSCRIPT}

me a plan of what to do, and he hadn't dealt with anybody with MS before and actually it was a bit of a disaster... (FG3:1).

Building a rapport with knowledgeable exercise specialists who were able to adapt exercises to take account of physical limitations and provide knowledge about safe and effective activities and appropriate progression was important: “... this was an opportunity for me to come to someone, a bit like a personal trainer really, who would know me, get to know me and teach me how to do it. Which is exactly what I got. For me that's what kept me coming..." (FG5:2). Participants felt that the exercise specialists taught them how to recognise signs that they are doing too much and how to pace themselves: "You are scared because you immediately get the symptoms from the increased body temperature and everything anyway, my feet automatically have pins and needles all up my legs and that is murder and it is a sign that I will have to stop and in actual fact what I have learnt is that it will fade, that is alright, it is your body just reacting and increasing temperature and is perfectly normal and carry on" (FG6:5). Participants described a shift in their perceptions by learning that they do not need to exercise to exhaustion to experience health benefits, and that they could progress slowly, at their own pace: "Whereas I wanted to run she was still teaching me to walk, so from that point of view I learnt a huge amount and gained a huge amount and that's how I am taking it forward. Not pushing it to the limits where I'm dropping, but building it up very slowly” (FG5:20) and “... you think I can't possibly do that and it just seems a huge obstacle but actually now knowing that you can do a

177 few minutes or a minute even at times and it will count, so it is achievable and attainable isn't it?" (FG6:2). Another participant said: "For me, I think it was taking the mystery out of exercise and giving the confidence that it's safe to do this and you'll get benefit from it... and I think guided exercise in the initial stages was the key" (T3). 
184 There were new insights in to the positive physical, mental and psychosocial experiences that engaging in an exercise program can bring. The majority of participants were grateful to have had the opportunity to take part in a tailored supervised exercise program; they recounted: “... I was looking forward to it every week, it gives you a regime to work to" (FG1:1) and "I just really enjoyed it and found it really rewarding” (FG6:2). In some cases, having a reason to 189 leave the house was a valued benefit of taking part in the program: "I really enjoyed the social aspect of meeting people and talking because you don't always get that when you have MS. You tend to be at home a lot on your own so I enjoyed coming...” (FG6:3). For others, improved health and fitness created new opportunities to engage in recreational activities with their families: "I try harder. My little boy will be surprised when I say yeah, alright I'll go swimming or yeah OK let's go for a walk, and he'll think Oh, that doesn't sound quite right" (FG4:4).

There were perceived improvements in physical fitness: Comments included: “...I think I felt generally stronger" (T2) and "I know for me, I felt I was a lot straighter, standing straighter" (FG1:2). Daily physical functioning was also improved: "I do find simple things like turning over in bed, which isn't a very easy task, and picking things up off the floor without collapsing; things of that nature are a lot easier" (T1) and there were reduced feelings of fatigue: "I feel as though I'm less fatigued. I don't have as many floppy days. I still get the odd one but nowhere near like it was" (FG4:7). The acute health benefits of participating in the exercise sessions 203 were also reported, including mood enhancement and a sense of wellbeing and achievement.

204 One participant recounted: "I always feel better having done the exercise" (FG1:4) and others spoke of: “A good sense of wellbeing and you'd achieved something” (FG3:1) and “...an adrenaline buzz, whilst you're doing it, so you feel that you've achieved something...” (FG3:4).

207 One participant reported finding the exercises difficult and at times experiencing some pain but 

her.

Theme 4: Perspectives on exercise adherence

212 Participants universally agreed that maintaining self-directed exercise at home was more

213 challenging than attending supervised sessions. Regarding adherence to the supervised sessions,

214 one participant said: “...I am the kind of person that will stick to an arrangement I've made. I

215 will stick to a commitment" (FG6:1). However, finding personal motivation to exercise and

216 having insufficient self-control to put their exercise intentions into action were barriers to

217 staying active at home: "I've found that because you have no structure once you are at home you

218 do tend to sit around and watch a bit more TV rather than when you have to come [to the

219 centre]... when you're at home, you think I'll not bother today" (FG6:3) and "I've had the incentive to come, and I've enjoyed coming, doing it at home and now it's finished I've just gone back to how it was before" (FG1.1).

223 For some, enjoyment of participating in exercise enhanced their motivation: "I've tried to keep

224 the willpower in my head... and it's working because I do enjoy it” (T4). For others, the 225 rationale for doing exercise at home was promoted by feelings of guilt: "Being at home I must admit I got a bit of a guilty feeling knowing that I was supposed to be doing another two

227 physical activities and thinking you can't just sit there, you've got to go out and do something.

228 There was a guilt thing that made me do the home [exercise], which I did, but also my husband

229 who was saying "don't you think you should be doing..." I got pressure from him which was 230 good but I think for enjoyment I much preferred [attending the centre] for the structured 231 [exercise]" (FG5:1). 
DISCUSSION

235 This qualitative study provides new insights into the exercise perspectives and experiences of 236 PwMS before, during and after participation in a program that was designed to develop the 237 confidence and skills for self-directed exercise. Following their MS diagnosis, many participants 238 experienced a loss of confidence in relation to their perceived physical capabilities and lack 239 knowledge of how to safely engage in exercise (Theme 1). Fear of exacerbating MS symptoms 240 and/or making the condition worse has previously been identified as a barrier to exercise in 241 PwMS 6, 10,20 and is compounded by a lack of MS-specific exercise advice (or conflicting 242 advice) following diagnosis (Theme 2). ${ }^{4,8,21,22}$ Our results reaffirm the need for confidence 243 building and MS-specific exercise advice following diagnosis.

245 In accordance with previous studies, ${ }^{6,7,13}$ participants felt that the supervised component of the program, and particularly having access to competent health professionals (exercise physiologist and physical therapist), was important for developing confidence to exercise via an improved awareness of their physical capabilities (Theme 2). Fatigue has been identified as an important

249 barrier to exercise in PwMS, ${ }^{10,21,23}$ and the importance of learning how to properly pace

250 themselves during exercise to avoid fatigue was valued, as was expert advice to progress slowly,

251 even if some found this frustrating. Through this reassurance, some participants learned not to 252 be afraid of the neurological sensations induced by exercise. Indeed, many experienced 253 significant health benefits from what they felt was a surprisingly low but manageable level of 254 physical activity. The average amount of weekly moderate intensity aerobic exercise achieved 255 by participants was $\sim 68$ minutes, ${ }^{15}$ representing only $\sim 45 \%$ of current recommendations. ${ }^{24}$

257 The reported improvements in physical functioning and perceptions of MS fatigue were 258 consistent with previous reports, ${ }^{3,22,25}$ but there were some new insights into how exercise can 259 improve everyday posture and daily functioning, from being able to stand more erect to an 
enhanced ability to reach down for things on the floor (Theme 3). The positive shift in perceived physical health and mental outlook also created new opportunities to enjoy more recreational physical activity time with their families. Acute mood enhancements and a sense of accomplishment during and after each exercise session were also experienced, as in previous

264 studies. ${ }^{14,21}$ Other participants placed great value on the opportunity for social interaction that 265 was presented by engaging in the exercise program, consistent with the findings of Learmonth et $266 \mathrm{al}^{26}$ especially where their MS had led to greater social isolation. These new insights highlight 267 the value of qualitative investigations in identifying health and psychosocial benefits associated 268 with exercise that are valued by PwMS.

270 Participants universally agreed that maintaining self-directed exercise during and after the 12-

271 week program was challenging, despite their positive experiences of exercise and newfound

272 knowledge gained from personally-tailored support (Theme 4). Many emphasised the

273 importance of attending supervised exercise sessions for maintaining their motivation for

274 exercise. Honouring pre-arranged appointments (related to supervised exercise), the enjoyment

275 of exercise and feelings of guilt were reported as important facilitators for self-directed exercise.

276 Conversely, the lack of weekly structure when supervision was withdrawn was identified as an

277 important barrier to self-directed exercise. Systematic review evidence shows that level of

278 contact with healthcare professionals, in addition to the use of behaviour change techniques (e.g.

279 goal-setting, self-monitoring) and engaging social support networks are important elements for

280 the maintenance of exercise behaviour change. ${ }^{27}$ In this respect, the effectiveness (and cost-

281 effectiveness) of novel approaches to maintaining contact with a knowledgeable heath

282 professional (e.g. provision of frequent face-to-face motivational booster sessions; use of mobile

283 and/or internet-based technologies to maintain contact, etc.) is an avenue for further research.

284 Peer-support and camaraderie with other exercising PwMS has been highlighted as another 
important facilitator for exercise amongst PwMS in previous research ${ }^{4,21}$ and a greater emphasis on helping PwMS to develop supportive social networks is also warranted.

\section{Study limitations}

289 An important limitation of this study is that participants were recruited from a single centre 290 serving a large catchment living within a few miles of the treating hospital. Hence, views regarding the importance of attending supervised exercise sessions may not be representative of PwMS from rural catchments living further away from appropriate MS-specific exercise classes. It is also possible that participants recruited for this study were generally more motivated to

294 engage in exercise than PwMS from the broader population, as they had all volunteered for the 295 intervention study. Additionally, only participants with mild to moderate MS were involved in 296 this qualitative investigation, and further research is needed to understand the challenges and benefits of exercise participation in PwMS who have higher levels of disability.

\section{CONCLUSIONS}

300 Our participants experienced a transition to inactivity following their MS diagnosis, compounded by a lack of MS-specific exercise advice across clinical and community settings. The individually-tailored support they received from knowledgeable exercise and physical therapy instructors during participation in the program was highly valued. However, the challenge of self-directed exercise and importance of continued motivational support for longterm maintenance was clearly evident in the views of our participants. Further research aimed at developing cost-effective strategies for establishing this provision is warranted. 
312 1. Asano M, Dawes DJ, Arafah A, et al. What does a structured review of the effectiveness of exercise interventions for persons with multiple sclerosis tell us about the challenges of designing trials? Mult Scler. 2009;15(4):412-421.

2. Motl RW, Gosney JL. Effect of exercise training on quality of life in multiple sclerosis: a meta-analysis. Mult Scler. 2008;14(1):129-135.

3. Rietberg MB, Brooks D, Uitdehaag BM, et al. Exercise therapy for multiple sclerosis. Cochrane Database Syst Rev. 2005(1):CD003980.

4. Aubrey G, Demain, S. Perceptions of group exercise in the management of multiple sclerosis. Int J Ther Rehab. 2012;19:557-565.

5. Borkoles E, Nicholls AR, Bell K, et al. The lived experiences of people diagnosed with multiple sclerosis in relation to exercise. Psychol Health. 2008;23(4):427-441.

6. Brown C, Kitchen K, Nicoll K. Barriers and facilitators related to participation in aquafitness programs for people with multiple sclerosis: a pilot study. Int J MS Care. $2012 ; 14(3): 132-141$

7. Christensen ME, Brincks J, Schnieber A, et al. The intention to exercise and the execution of exercise among persons with multiple sclerosis--a qualitative metasynthesis. Disabil Rehabil. 2016;38(11):1023-1033.

8. Learmonth YC, Adamson BC, Balto JM, et al. Multiple sclerosis patients need and want information on exercise promotion from healthcare providers: a qualitative study. Health Expect. 2016.

9. Plow MA, Resnik L, Allen SM. Exploring physical activity behaviour of persons with multiple sclerosis: a qualitative pilot study. Disabil Rehabil. 2009;31(20):1652-1665. physical activity for people with multiple sclerosis: a qualitative investigation. Disabil 


\section{ACCEPTED MANUSCRIPT}

11. Klaren RE, Motl RW, Dlugonski D, et al. Objectively quantified physical activity in persons with multiple sclerosis. Arch Phys Med Rehabil. 2013;94(12):2342-2348.

12. Dlugonski D, Joyce RJ, Motl RW. Meanings, motivations, and strategies for engaging in physical activity among women with multiple sclerosis. Disabil Rehabil. 2012;34(25):21482157.

13. Dodd KJ, Taylor NF, Denisenko S, et al. A qualitative analysis of a progressive resistance exercise programme for people with multiple sclerosis. Disabil Rehabil. 2006;28(18):11271134.

14. Kasser S. Exercising with multiple sclerosis: insights into meaning and motivation. Adapt Phys Activ Q. 2009;26(3):274-289.

15. Carter A, Daley A, Humphreys L, et al. Pragmatic intervention for increasing self-directed exercise behaviour and improving important health outcomes in people with multiple sclerosis: a randomised controlled trial. Mult Scler. 2014;20(8):1112-1122.

16. Tosh J, Dixon S, Carter A, et al. Cost effectiveness of a pragmatic exercise intervention (EXIMS) for people with multiple sclerosis: economic evaluation of a randomised controlled trial. Mult Scler. 2014;20(8):1123-1130.

17. Fosnot CT. Constructivism: A psychological theory of learning. In Fosnot CT, (Ed). Constructivism: Theory, perspectives, and practice New York: Teachers College Press 1996:8-83.

18. Gale NK, Heath G, Cameron E, et al. Using the framework method for the analysis of qualitative data in multi-disciplinary health research. BMC Med Res Methodol. 2013;13:117.

19. Pope C, Ziebland S, Mays N. Qualitative research in health care. Analysing qualitative data. BMJ. 2000;320(7227):114-116.

20. Clarke R, Coote S. Perceptions of Participants in a Group, Community, Exercise Programme for People with Multiple Sclerosis. Rehabil Res Pract. 2015;2015:123494. 


\section{ACCEPTED MANUSCRIPT}

21. Learmonth YC, Motl RW. Physical activity and exercise training in multiple sclerosis: a review and content analysis of qualitative research identifying perceived determinants and consequences. Disabil Rehabil. 2016;38(13):1227-1242.

22. Markwick R, Singleton C, Conduit J. The perceptions of people with multiple sclerosis about the NHS provision of physiotherapy services. Disabil Rehabil. 2014;36(2):131-135.

23. Horton S, MacDonald DJ, Erickson K, et al. A qualitative investigation of exercising with MS and the impact on the spousal relationship. Eur Rev Aging Phys Act. 2015;12:3.

24. Chief Medical Officers. Department of Health PA, Health Improvement and Protection. Start Active, Stay Active: A report on physical activity for health from the four home countries'. 2011.

25. Andreasen AK, Stenager E, Dalgas U. The effect of exercise therapy on fatigue in multiple sclerosis. Mult Scler. 2011;17(9):1041-1054.

26. Learmonth YC, Marshall-McKenna R, Paul L, et al. A qualitative exploration of the impact of a 12-week group exercise class for those moderately affected with multiple sclerosis. Disabil Rehabil. 2013;35(1):81-88.

27. Greaves CJ, Sheppard KE, Abraham C, et al. Systematic review of reviews of intervention components associated with increased effectiveness in dietary and physical activity interventions. BMC Public Health. 2011;11:119. 
Table 1. Characteristics of the participants

\section{Characteristics}

Focus group participants $(\mathrm{N}=29)$

Females ( $n$ ) $23(79 \%)$

Age (y) $48.8 \pm 8.2$

Years since MS diagnosis

$8.8 \pm 7.0$

EDSS

$3.8(1.0-6.0)$

Interview participants $(\mathrm{N}=4)$

Females ( $n)$

$3(75 \%)$

Age (y)

$48.8 \pm 7.3$

Years since MS diagnosis

$9.7 \pm 3.5$

EDSS

$3.0(1.5-6.5)$

Age and years since MS diagnosis are presented as mean \pm SD. Expanded Disability Status Scale (EDSS) is presented as median (range). 
Table 2. Semi-structured "a-priori" topic guide used to guide discussions

\section{ACCEPTED MANUSCRIPT}

\section{Section 1: Exercise experience before the EXIMS trial}

Can you tell us if prior to your diagnosis, whether you took part in exercise?

How did you find this experience?

How has this changed since your diagnosis?

Have you been given advice in the past from health professionals regarding exercise?

Can you tell us who gave you the advice and what the advice was?

\section{Section 2: Exercise experience during the EXIMS trial}

Can you tell us about your exercise experiences at the University; was it difficult at the beginning? Did it get any easier? At what point was that?

How did you feel during and after the sessions?

What kept you coming to the exercise sessions?

Did you like the structure of the exercise sessions?

- Was it the right amount?

- Did you like the mixture of home and supervised exercise?

- Did you like the gradual reduction in contact time?

Did the intervention, in any way help you to become more active? Can you explain?

Have you any suggestions for how the exercise might be improved?

Did you notice any improvements/benefits from the exercise? If so what?

Did you notice any effects on your symptoms? fatigue, pain, sleep etc.

\section{Section 3: Exercise after the EXIMS trial}

Have you remained physically active since completing the study?

Did the supervised exercise sessions at SHU provide you with any knowledge/skills that you have been able to put into practice following the trial?

Do you feel that you are more physically active now than before the trial and in what way?

Has the intervention had any influence on your confidence to exercise?

Can you give an example?

What things stop you from participating in activity/exercise?

Is there anything that you feel we could have done during the trial to assist you in remaining more active?

What help if any do you feel you need to stay active?

Would you recommend exercise to others with MS?

Are there any other comments that you would like to make? 